Article

\title{
Effect of Information System Artifacts on Organizational Resilience: A Study among Malaysian SMEs
}

\author{
Shubashini Rathina Velu ${ }^{1}$, Abdullah Al Mamun ${ }^{2, *}$, Thavamaran Kanesan ${ }^{3}$, Naeem Hayat ${ }^{2}$ \\ and Sharmini Gopinathan ${ }^{1}$ \\ 1 Faculty of Management, Multimedia University Persiaran Multimedia, 63100 Cyberjaya, Selangor, Malaysia; \\ shubashini.rathina@mmu.edu.my or shuba.rv@gmail.com (S.R.V.); \\ sharmini.gopinathan@mmu.edu.my (S.G.) \\ 2 Faculty of Entrepreneurship and Business, University Malaysia Kelantan, Karung Berkunci 36, \\ Pengkalan Chepa, Kota Bharu 16100, Kelantan, Malaysia; naeem.a18e013f@siswa.umk.edu.my \\ 3 Academic Consultant, Research and Publications, Cyberjaya 63000, Malaysia; \\ thava@proofreadingbyphd.com \\ * Correspondence: abdullah.a@umk.edu.my or mamun7793@gmail.com; Tel.: +133003630
}

Received: 12 May 2019; Accepted: 3 June 2019; Published: 6 June 2019

\begin{abstract}
Conducting business nowadays has become much more challenging, and the importance of collective behavioral capacity is paramount regarding the behavior of employees along with internal information systems that form the backbone of many organizations in terms of competitiveness and survivability. This study, therefore, examined the effect of commitment, communication, competency, community, connection, consideration, and coordination on information system artifacts and the effect of information system artifacts on organizational resilience among Malaysian SMEs. To verify these statements and positioning, a sample of employees from registered organizations of the Malaysian Digital Economic Corporation Sdn Bhd (MDEC), participated in this study to examine a number of aspects. Quantitative data were collected from a total of 252 respondents through a questionnaire survey and data were analyzed using PLS-SEM. We performed the endogeniety assessment for the all the seven input variables and confirmed lack of endogeniety issues. The findings of this study constitute essential results that the commitment, communication, competency, community, connection, consideration, and coordination positively influenced the ISA. Furthermore, the mediating effect of ISA on the relationships of organizational resilience with commitment, communication, competency, community, connection, consideration, and coordination, had been revealed. The study concluded that a positive set of behavioral capital, if it exists inside organizations, enables the organizations to prosper and survive. Similarly, policymakers need to address the issue surrounding resilience in SMEs by establishing the correct benchmarking mechanism by introducing it as a formal activity to be undertaken regularly within the SMEs. Given the vast majority of employment in Malaysia and globally is provided through SMEs, if they become more resilient to change and to unforeseen events, then the employment of the vast majority of the working class can be secured across different nations.
\end{abstract}

Keywords: collective behaviors; information system artifact; organizational resilience; SMEs

\section{Introduction}

Present day business organizations, whether globally or locally continue to be challenged through competition and external market forces requiring the business to adjust through making changes to operating procedures and processes in order to remain competitive and survive. Although, while business survival is the prime objective, in this case, resilience and perseverance and are equally 
important. Resilience can be described as the capacity to utilize internal resources, such as people and processes within an organization in dealing with uncertainty and misfortune and accomplishment. Mallak and Yildiz [1] revealed that organizational resilience is the most important attribute of modern organizations to remain competitive and likewise, organizations with poor resilience may not survive into the next decade. Therefore, organizational resilience is the capacity that can be developed and maintained at the organizational level through utilizing resources at the disposal of the organization [2]. From a developmental viewpoint, it is a means to manage, develop, and maintain the much-needed capacities to ensure the organization is resilient [3]. This also facilitates the progression of the organization at the national level by helping to created job opportunities. In this study, the capacity viewpoint concerning organizational resilience is premised on the organizational internal collective perception of employees through their commitment, communication, connection, coordination, community, consideration, and competency working through the information system artifact (ISA) of the organization. Additionally, this study aims to provide empirical support to enhance the understanding of organizational resilience through measuring capacity and future enhancement.

The study of organizational resilience has attracted the interest and attention of many researchers involved with SMEs [3]. In Malaysia, the resilience of SMEs is especially important given this sector employs the vast majority of workers. This is similar at the global level as well [4]. Likewise, a significant number of Malaysian SMEs have adopted technology, including information systems to efficiently operate their business ventures. Although, in the context of SME's and the role of ISA this area is relatively unexplored [5]. As briefly mentioned earlier, SMEs comprise of employees who are utilized as resources in supporting the business along with information systems [5,6]. These two resources from a development perspective, enable the SME to become resilient to unforeseen changes or uncertainty. Malaysian SMEs are acknowledged as having deficient skills and knowledge, and a lack internal teamwork in the form of communication, community, consideration, and competency which are needed to generate commitment, connection, and coordination within the organization [3], via the ISA [5]. Accordingly, it is important to determine the importance of communication, community, competency, commitment, consideration, connection, and coordination with the ISA that helps to enable SMEs to achieve resilience.

The collective employee capacities of commitment, communication, competency, connection, coordination, and community mediated by the ISA utilized in Malaysian SMEs are examined in the context of this study. The collective employee capacities and the ISA work together in order to create resilience for the SME to sustain the business [7]. These capacities, along with information systems, can be developed and integrated over time and customized to coincide with the changing environment and other internal factors [3]. However, the perception of consideration as a capacity factor has the adverse effect on the ISA given that the majority of SME employees are not conversant nor clear about the objectives or application of the ISA [8]. This, therefore, prompts management to consider the matter and take necessary action [9]. The design and implementation of the ISA must be carried out with the involvement of employees, and necessary training must be provided to support the realization of the benefits in facilitating organizational resilience. Limited endogeneity issues were found to be associated with all seven input variables from the endogeneity assessment. Therefore, management scholars need to understand and address the issue of endogeneity that may arise in SME related studies resulting from SME culture as well as from market forces. Notably, endogeneity was not discussed in the literature as a prominent causality issue with respect to organizational resilience.

The following section presents the literature regarding organizational resilience and ISA, and debate surrounding the measurement of organizational resilience and ISA. The method adopted in this study is based on reviewing the literature and development of hypotheses. The analysis and results are discussed in Sections 4 and 5 followed by Section 6 which presents the overall conclusions of the study along with future research opportunities and limitations of the study. 


\section{Literature Review}

The notion surrounding resilience originated from the area of bio-science and has been utilized in the field of psychology in explaining the capacity of human beings to deal with the pressure of life and improve the capacity to be resilient in order to survive $[7,10]$. Resilience can be displayed in certain events such as work, personal experience, or disaster situations [11]. Individual resilience rests on personal life experiences in facing difficult times or situations that the individual faced in his/her lifetime [7]. Individuals mainly engage in resilience behavior when they experience difficult or troublesome situations such as where their survival is at risk; portrayed as protective and lifesaving behaviors $[7,10]$.

Similarly, the perception of risk is highly related to the characteristic features of individuals [12]. In this sense, risk factors within and around the surrounding environment require the right attention and behavior. In other words, displaying the right behavior depends on the adaptive capacity of the individual to respond to the risky situation at hand [13]. Although individuals differ in their capacity to adapt to risk, this often leads to exhibiting different behaviors towards challenging situations based on the differences in their adaptive capacity and recognition towards risk. Indeed, this is the main reason that the resilience behavioral model is based on the perception of vulnerability [2].

\subsection{Organizational Resilience}

Organizations can be described as living entities having three characteristic features, namely purpose, people, and structure. Historically, organizational history is full of failures and few organizations nowadays older than 100 years remain operating. Organizational resilience was studied by Horne and Orr [7], as the capacity of the organization to survive whereby the viewpoint concerning capacity and its relationship to organizational resilience stimulates the development mindset concerning organizational resilience. On the other hand, Luthans [14] (p. 702) delineates resilience as "the developable capacity to rebound or bounce back from adversity, conflict, and failure or even positive events, progress, and increased responsibility".

Therefore, resilience is the innate capacity of the organization to face adversity, conflict, and even failure but subsequently bounce back from these problematic situations and progresses by moving forward which is considered the responsibility of the organization. The behaviors associated with facing difficult situations or adversity enacts the responsibility of the organization to revert to business as usual to satisfy its stakeholders. This responsibility behavior, in turn, triggers the developmental mindset in the organizational community and for those organizations engaged in the learning process. Importantly, this enables organizational learning phenomenon to learn from internal situations and from external factors in building the capacity to face adversity in maintaining business as usual [10]. This organizational learning posture learned by the organization leads inevitably into a learning organization [15]. Furthermore, the learning organization framework stimulates or prompts the organization to learn from all aspects and experiences and across all organizational levels. Indeed, this enables the organization to learn, from an enterprise-wide perspective and not just at one stage of organizational learning [15].

A study by Mallak [16] considered the threat conditions in an organizational environment and how the organization responds to these challenges in order to survive. It is a normal propensity for an organization to manage risk or challenges and return to normal operations following the passing of a risky or troublesome situation [17]. However, organizational responses to threats and challenging situations form part of the overall business strategy based on the ideology of the organization [2]. In other words, organizational resilience is the capability of the organization to manage internal and external problems [1]. It is also believed that both conceptualizations of organizational resilience have similarities given that both emphasize organization survival or dealing with challenging issues and returning to normal operating conditions.

Similarly, another perspective of organizational resilience as being intrinsic and unique is shared by [11], Hollnagel [18] as the hidden aptitude of the organization by Powley [19]. Whereas, Gilly, 
Kechidi, and Talbot [20] labeled organizational resilience as the dynamic and responsive ability of an organization. In a separate study, organizational resilience is portrayed as being both incremental and vigorous [21]. Accordingly, this highlights that organizational resilience cannot merely be obtained at one time but rather is a continual process of moving forward, collecting experiences along the way and to attain greater resilience and ability to face the many uncertain challenges presented [22]. Furthermore, changes and advancements in technology —in the industry and globally-also present organizational challenges [23]. Importantly, an organization will not survive without the ability to be resilient in facing adversity.

Notwithstanding, given the changing nature of work, the work environment and global economic and political conditions, organizational survival at any level remains challenging. McManus et al. [12] assert that resilience is the amalgamation of adaptive capacity, situational awareness, and the management of vulnerability at the organizational level. Whereas, adaptive capacity is the ability of the organization, as a system, to adjust according to changing environmental factors [24]. The system concept posits that an organization will be faced and impacted by many factors ranging from internal factors to external environmental factors. Therefore, organizations need to build the ability to make the necessary adjustments in order to survive according to the factors that are impacting them [21].

Moreover, the ability to be more resilience is associated with the awareness of the factors impacting the organization. In this sense, internal capacity is crucial for the effective management of troublesome situations that may impact the organization. Here, flexibility, adaptability, agility, and efficiency are common attributes in enabling organizational resilience [25]. Although, organizational resilience is a function of the organization's adaptability, responsiveness, sustainability, and competitiveness [26]. One might presume that the ability concerning flexibility and providing an appropriate response is necessary in order to become resilient but maintaining a level of competitiveness is more important in order to survive [20]. Nonetheless, the capacity to survive is associated with the capability to internally respond to the external environment by trigging the responses that are needed to be resilient. It is understandable that organizational resilience is a multidimensional construct, mainly with an external focus, which is driven by external environmental cues [27]. Moreover, resilience is the ability to absorb unsavory events or risks to maintain business as usual.

Lengnick-Hall, Beck, and Lengnick-Hall [28] presented organizational resilience based on three aspects of organizational dimensions namely cognitive, behavioral and contextual constituents [29]. The cognitive aspect makes sense of reality, based on the employees perceived ideological association with the organization that builds on value toning, cognitive belief and organizational values delivered to employees $[14,30]$. These beliefs and values bring with it a citizenship attitude in the employees that enable the behaviors most demanded by the organization in order to work together as a community [15]. After achieving cognitive alignment, the notion of action or behaviors become much easier for employees to display and achieve resilient behavior [31]. Behavioral resilience can only be achieved when employees have a cognitive attachment [11]. The context, in this case, provides the necessary conditions to enable the proper behaviors to be enacted along with the proper values being displayed comparable to the social capital within the organization. For example, resources being shared by employees in the form of networks [15].

Nevertheless, Wicker, Filo, and Cuskelly [32] extended the understanding of two-dimensional organizational resilience from both the operational and strategic perspective. Here they posited that operational resilience at the organization level is an organizational aptitude to absorb shock and then returning to the normal organizational condition as before the shock occurred. Organizational resilience is multifaceted, and a multilevel phenomenon that builds on the interaction of the causes that exist internally within the organization and externally, prompting the necessary behavior or actions to occur in the form of resilience. Organizational resilience is based on the availability of organizational resources which are delivered in the form of operational behaviors directed by the organization's strategic mindset that creates a resilient organization. 


\subsection{Assessment of Organizational Resilience}

As mentioned earlier, the valuation of organizational resilience is multifaceted. Based on the suggestion of Mallak [16] and Somers [22] endorsed the estimation of organizational resilience based on seven distinct characteristics of organizational resilience, namely perceived experiences constructively perform with positive adaptive behaviors, adequacy of external resources, expansion in decision-making boundaries, practicing bricolage, tolerance for uncertainty, and building virtual role systems.

Furthermore, the resilience of an organization is based on four factors, namely situation awareness, management of keystone vulnerabilities, quality, and adaptive capacity [32]. Lee et al. [8] proposed the evaluation of organizational resilience applying a scale using certain factors along with 73 items. Whereas, Whitman, Kachali, Roger, Vargo, and Seville [33] posited a shorter version of the scale but using the same four factors proposed by Lee et al. [8] but with 52 items. Lee et al.'s [8] scale consisted of several factors representing innovation and creativity, collaboration similar to the scale of McManus et al. [12]. Moreover, that resilience is approximated using assets management, resilience requirement and development, risk management, people management, and monitoring at the operational level.

Another approach that can be employed is to base organizational resilience on the system theory which assumes that members of the organization are actual resources as well as the capacity of the organization to become a resilient organization [34]. The idea, in this case, is for the organization to have resilience; an organization needs resilient employees along with internal systems that activate or trigger organizational resilience. Accordingly, this leads to using an information system or artifact. A social system is also required to face potential threats and challenging situations aimed at the organization having an organizational system to respond to these challenges or conditions [30]. Consequently, the behavioral capacities of the employees can act as the enabler for organizational resilience [2,7], via the information system $[29,35]$. Furthermore, the resilience of the organization does not merely reside on organizational resources in totality but rather is a collaborative approach along with the joint effort of stakeholders. For instance, employees, suppliers, other market players, and policymakers [3] and the existing system that operationally performs well inside the organization [20]. Moreover, endogeniety was not discussed in the literature as a prominent causality issue in the organizational resilience.

\subsection{Information System Artifacts}

Activity theory postulates that the ISA provides the necessary activities and conditions to support an organization [29], used as tools that can assist employees in performing their tasks and activities more efficiently. An organizational informational tool facilitates and enables better comprehension relating to the activities for employees [29]. The ISA assists in generating and enhancing coherence within the workplace environment, apart from enabling the collective working amongst the employees where common meaning and sharing of information become easier. The ISA acts similar to an instrument which strengthens the action patterns thereby helping to create common sense concerning objectivity for the community at large $[25,26]$.

However, the ISA perception in the mindset of the user relies on the interaction between the user and the ISA. The ISA offers certain features that assist the worker in performing his/her tasks and connects the user with a common platform to interact with at the organizational level. The innovation within the organization also assists employees to improve their capacity and engagement. Accordingly, the ISA is not merely a static mix of features but is a dynamic bundle of user-driven needs and helps to fulfil certain user behaviors [5]. Consequently, this leads to the phenomenon of organizational resilience [3-5]. As the ISA creates awareness and effective response patterns, the use of technology can help to instill disaster cognition and the effective management of a disaster event [5].

In effect, the ISA operates like a platform in IT-driven organizations, where employees perform their daily activities and other duties as well as a tool to engage within the organization with other users and acts as a learning platform [25]. The ISA acts as a social actor in many respects and goes beyond the concept of a productivity-enhancing tool [10]. Moreover, this common platform helps 
to acquire the collective conscience of the organization at the organizational level. Indeed, it does not only reside as a helping/learning tool but also provides a dynamic working environment for all users connecting them like a community where they collectively work and interact as part of the community [6]. The collective community thereby leads to uniform behaviors at the organizational level that enables common behaviors of certain types to be enacted as expected and required by the organization $[12,25]$.

Given the common behaviors promoted by the ISA, collective competency begins to develop as the users of ISA start to perform similar tasks cohesively and collectively with each other as connected in the system [34]. As one completes his/her work/task, other work is then started, forming a progressive chain of events. The notion of coordination established and employees have a common meaning with community understanding enabling to achieve commitment. Accordingly, this generates the working system within the organization where the ISA connects with each other, communication becomes easier, and the ISA positively influences the level of commitment shared by employees $[2,25]$. Therefore, one can assume that the ISA creates an enabling environment and culture where each person working in the organization is in effect connected in performing daily tasks and purposely prepared for contingencies in the event of interruptions or change.

\subsection{Hypothesis Development}

In this section, the viewpoint of organizational resilience is regarded as having the capacity to be resilient; as cognitive, behavioral, and contextual characteristics that prompt the organization to have resilience [28]. Capacity in the form of resilience can be developed, managed, and stored within the collective efforts of the organization's employees [28]. Accordingly, this leads to exploring the possible role of the collective behavioral stock of employees towards organizational resilience that results through the ISA implemented in the organization. Also, the collective behavioral model of organizational resilience is contemplated. Organizational resilience is a collection of six modules, such as vision, values, elasticity, empowerment, coping, and connections [16].

Subsequently, the work of Somers [22] grounded on public organizations, tests Mallak's [16] structure by examining individuals as well as examining organizational resilience objectives. The work acknowledged that organizations are a social organism in form, comprising of people, structure, and objectives that guide the organization. Furthermore, the good or bad operations of an organization rest on the action of people and responding to the internal and external challenges faced by the organization from time to time. This ability to react and counter in order to revert to the previous situation following the uncertain situation and circumstance is regarded as the concept behind a resilient organization [21].

Nevertheless, this capability to combat or revert to business as usual, is unassociated with waiting for contingencies to occur or challenges to come along, but more importantly, that organizations receive the right information in order to reduce the level of vulnerability more efficiently through the ISA [2,25]. Indeed, this makes an organization resilient which provides a strong argument for collective sensemaking and working with shared objectives with collective capacities to achieve organizational goals through the implantation of the ISA. This aggregation of behaviors is also objectively mentioned by Lengnick-Hall et al. [28] and the use of the ISA for resilience [25].

\subsubsection{Collective Commitment}

As employees are agents of the organization [36], therefore employee behaviors embody organizational activities [14]. Committed employees are the most significant and highly valued resource in any organization as they work towards the best interests of the organization [36]. The collective perceived commitment portrays the general sense of motivation that prevails in the organization. Employees are willing to work with each other and take charge when necessary upon having ownership of work-related tasks for routine work or unexpected events. This sense of collective commitment is further enhanced when employees have a common information system platform. In other words, 
the commitment of employees as described and charged by the common workplace is shaped by the ISA [25]. Therefore, the following hypothesis is posited:

Hypothesis 1 (H1). The collective sense of commitment has a positive effect on the information system artifacts.

\subsubsection{Communication within the Organization}

Communication is the process of exchanging ideas and information associated with the living organism. Organizations, as a form of social system communication, enable the organization to operate for collective causes along with collective efforts. Likewise, information accuracy and precision leads to knowledge that is essential for the performance of organizational tasks and in the understanding of environmental issues [12]. Moreover, communication enables the cultivation of openness and shared knowledge, where employees feel a sense of belonging which is an important component of any social system that exists inside an organization [9]. Organizational communication is a vital part of the culture as well as the prevailing norms of the organization [2]. When communication is supported and facilitated through the use of the ISA, communication becomes more effective as the common medium within an organization, given it is in the shape of the ISA. Moreover, the ISA stores the information and work as a learning tool for the future [25]. The above discussion, therefore, leads to the following hypothesis:

Hypothesis 2 (H2). The collective sense of communication has a positive effect on the information system artifacts.

\subsubsection{Collective Community}

The flow of information within an organization leads to the lessening of ambiguity among organizational employees so that employees can perform organizational tasks efficiently and correctly. Moreover, employees feeling greater contentment within the organization will extend their efforts further in resolving organizational problems [8]. Similarly, employees with a team-based attitude and approach will work in one direction to manage and overcome organizational disruptions in order to overcome any crisis. The use of an information system undoubtedly helps to inspire a community feeling among organizational employees [25]. Accordingly, the connectivity that employees experience through the provision of the ISA makes employees feel like part of the community. Therefore, the following hypothesis is presented:

Hypothesis 3 (H3). The collective sense of community has a positive effect on information system artifacts.

\subsubsection{Collective Competency}

Employees bring their competencies to the workplace which helps the organization to perform. In fact, the organization is the collection of collective competencies of employees [28]. When all individual capacities are at the disposal of the organization, the capacity to look forward to opportunities and threats is consequently multiplied. These collective competencies help in making an organization have a purpose and with actions linked in a more conducive and appropriate manner [9]. The ISA also works as the competency register, capturing every aspect of the employee's skills, knowledge, and experiences [25]. Accordingly, the collective competency is well managed through the assistance of the ISA. Therefore, the following hypothesis is presented:

Hypothesis 4 (H4). The collective sense of competence has a positive effect on information system artifacts. 


\subsubsection{Collective Connectedness}

While organizations are a collection of people and interrelationships, they also vary concerning these relationships. A healthy organization will be associated with having high interrelationships and strong ties/connections [6]. However, an organization having weak interrelationships and loose ties/connections is considered a weak organization. Consequently, this collective connectedness represents organizational resilience [13]. Furthermore, having a connection outside of market players is also important for organizational resilience [12]. Nowadays, people are more connected due to information systems, the internet, and mobile devices which is also true for organizations as well. Enterprise-wide information systems act like a common workplace that connects employees, enabling them to work more cohesively with a team-based attitude [25]. Therefore, the following hypothesis is posited:

Hypothesis 5 (H5). The collective sense of connectedness has a positive effect on information system artifacts.

\subsubsection{Collective Coordination}

Coordination is the essential ingredient needed to sustain an organization and also for the organization as a system, to accomplish organizational goals and objectives. Indeed, this coordination is reflected and represented through the organizational structure that enables the organization to operate as a complete system [12]. Collective efforts cannot bring about positive outcomes or change if work efforts are uncoordinated [9]. Importantly, coordination brings with it, the necessary conditions for the organization to work in teams to successfully achieve assigned tasks and to analyze the susceptibilities of certain situations that may adversely affect the organization [12]. Coordination enriches organizational life through the division of tasks and achievements. Tasks can be assigned or allocated more efficiently which enriches the organization by using the information system [25]. The information system empowers the coordination in real time and allows work division and team formulation to occur via virtual teams to complete tasks in the best way [1,34]. Therefore, the following hypothesis is presented:

Hypothesis 6 (H6). The collective sense of coordination has a positive effect on information system artifacts.

\subsubsection{Collective Consideration}

Collective consideration is about priority setting inside the organization [9]. Consideration provides the necessary direction and conditions in order for the organization to achieve the desired outcomes concerning the collective behaviors of employees. Importantly, direction helps to provide the necessary support that is needed, thereby establishing the necessary conditions or state needed instead of forming a negative or a discouraging mindset. Consideration provides the motivation to move forward for an organization during times of crisis through self-evaluation in order to improve the situation when it next eventuates. This is especially important for employees [24]. Resilience can also be achieved using the ISA, given it provides the system and support necessary to revert to business as usual. The information system also enables in having the right information readily available to achieve resilience and decision making as well as a mechanism to learn for future situations and events [25]. Accordingly, the following hypothesis is presented:

Hypothesis 7 (H7). The collective sense of consideration has a positive effect on information system artifacts. 


\subsubsection{Information System Artifacts and Organizational Resilience}

Through the ISA, maintaining and storing information, tracking, and learning from past events becomes much easier. Although, it also helps to have a sequential and logical framework in place to ensure the information is readily available for use within the organization. The ISA works for the cognitive as well as the behavioral part for employees in providing better working conditions which help to achieve integration at the workplace level and reducing inflexibility inside the organization [21]. Indeed, this results in encouraging a team-based environment in that employees have a common purpose in working within the organization as one cohesive unit [10]. In this case, the implementation of the ISA provides the necessary conditions and infrastructure to achieve resilience that an organization is seeking to achieve concerning the availability, accuracy, and timeliness of information and in allocating tasks [25]. Moreover, the ISA helps to achieve progress, by moving forward which facilitates a learning environment to be created based on previous mistakes and experiences in helping and guiding the organization to handle future challenges. Therefore, the following hypothesis is proposed:

Hypothesis 8 (H8). Information system artifacts have a positive effect on organizational resilience.

\subsubsection{Mediational Role of Information System Artifacts}

The ISA is primarily based on an activity concept where the implementation of the ISA positively affects employee capacity and engagement by enhancing the individual's resilience levels [25]. This study contributes to the resilience stream of knowledge by testing the role of the ISA as a mediator between the employee's perceived capacities gained through working in the organization with respect to the employee becoming a citizen of the organization and contributing to the resilience of the organization to face untimely challenges [10]. The ISA also acts like a learning platform that motivates employees, enabling them to interact in a more conducive manner [25]. The resilience of the organization is therefore based on the collective perceived behaviors of employees such as their commitment, communication, competency, connection, coordination, community, and consideration [9]. In this case, the availability of the ISA activates these behaviors to work in a conducive manner towards organizational resilience [25,37]. Importantly, organizational resilience relies on the collective perceived behaviors of employees and the capacities associated with the ISA available in the organization $[3,10,25,37]$. Accordingly, based on the above discussion and supported by the finding from the previous studies, the following hypotheses are presented:

Hypothesis 9 (H9). Information system artifact mediates the relationship between collective commitment, collective communication, collective perception, community, collective competency, collective coordination, and collective consideration with organizational resilience.

\section{Research Methodology}

A quantitative cross-sectional design approach was employed in this study to investigate the relationships between the employees' perceptions of commitment, communication, community, competency, connection, coordination, and consideration on organizational resilience mediated by the ISA. The data were collected from business organizations registered under MDEC, Malaysia. The population sample comprised of MDEC employees having working experiences associated with a team attitude, ISA, and with appropriate awareness of organizational resilience. All associations hypothesized and tested are presented in Figure 1.

\subsection{Sample Selection}

The sample size was calculated using G-Power version 3.1 software. The parameters were set at 0.95 , and the effect size was estimated at 0.15 based on the seven predictors for the outcome; the effective 
sample size was 153. The suggestions given for sample size in SEM must be at least 10 times that of the paths in the structure as well as the measurement model [38]. Therefore, as the sample size for this needed to be higher than 100, a sample size of 200 was chosen for the collection of responses. In total, 500 questionnaires were distributed to the selected 500 enterprises registered with MDEC. A total of 292 questionnaires were returned, with 252 being completed and usable for this study.

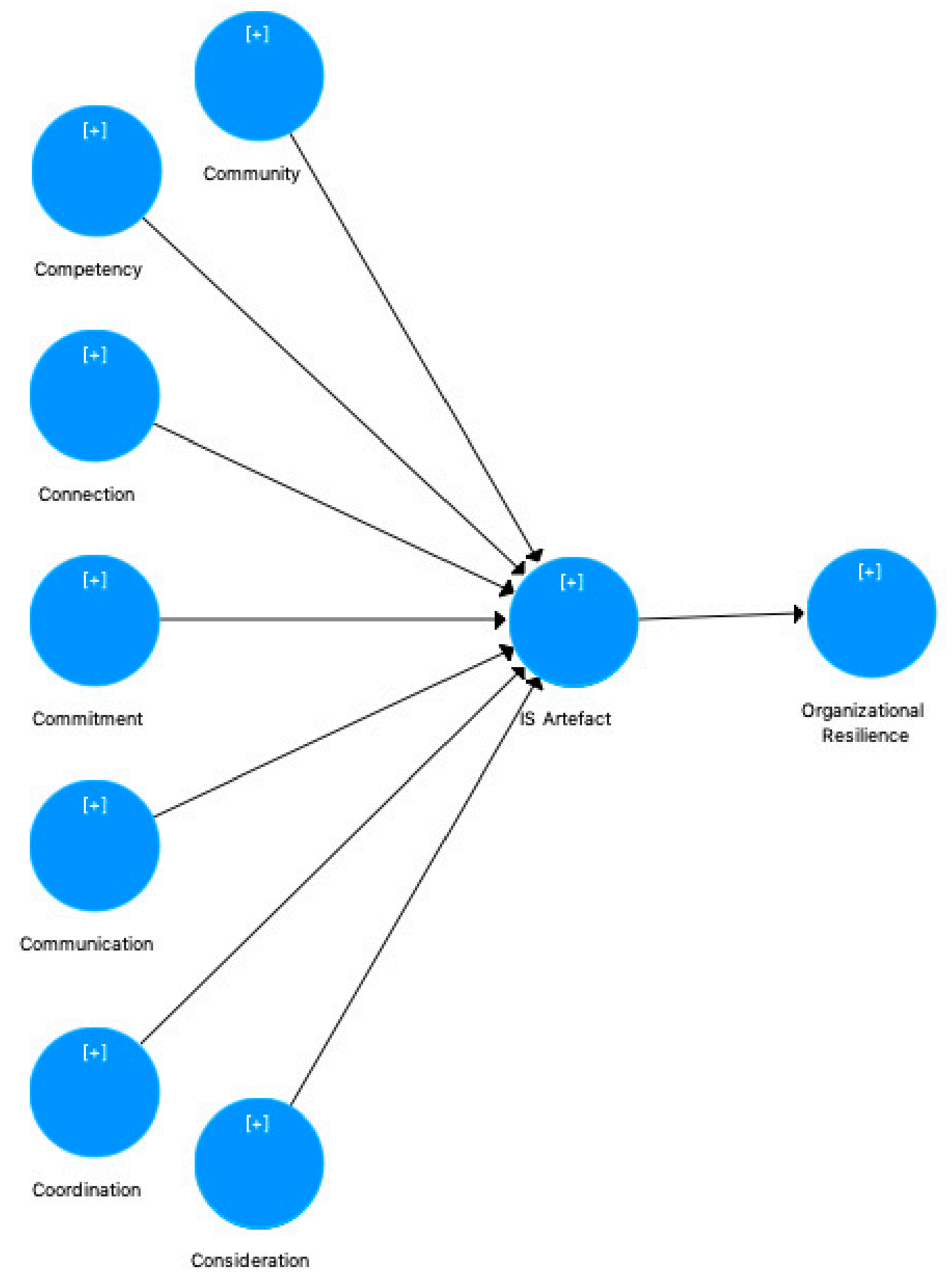

Figure 1. Research model.

\subsection{Research Instrument}

In order to acquire an acceptable research response, the scale of items used in the questionnaire needed to be reasonably straightforward and easy for respondents to comprehend. In this study, 
the items were adapted from previously developed scales found in the literature. Commitment at the workplace is revealed as an extensive area of research, and the work of Allen and Meyer [36] provided the necessary guidelines adapted in this study. Additionally, the work of McManus et al. [12] provided a rich understanding of the commitment that exists and supports the success of an organization. The perception of the commitment of organizational employees was estimated by applying five statements as next described.

Communication forms the heart and pulse of an organization. McManus et al. [12] and Connor and Davidson [9] investigated the importance of communication internally and externally of the organization. Moreover, communication as the behavior of the employee was based on five items in the sense that an organization can be a collection of people, in which they form a community inside the organization. Similarly, Lee et al. [8] and Gilly et al. [20] provided guidelines to understand and explore the role of the community within the organization which relates to team feel and like to work in a group environment. Here, five items were utilized to estimate the collective community perception within the organization.

The work of Connor and Davidson [9] provided the means to use collective competency as a resource that enables an organization to be resilient and deals with the collective competency to managing a crisis and taking responsibility to work with a sufficient level of competency. To estimate the collective competency of the employees five statements were utilized. Another area is the connection perception within the community of the organization. According to Connor and Davidson [9] and Burns et al. [5] they provided a practical suggestion to estimate the connection perception within the organization which related to having close ties/connections and working in teams for effectiveness. Also, to assess the connection between the organization's employees' five statements were used.

Similarly, the work of McManus et al. [12] and Connor and Davidson [9] explored the nature and importance of coordination within the organization. Here, coordination has the facets in identifying the issue, the differences and negotiation to achieve consensus. Here, in the estimation, five statements were employed. For the assessment of the consideration, the work of McManus et al. [12] and Connor and Davidson [9] was adopted in this study which included the organizational priority setting, response to the problems, having the right motivation, and informed decisions in providing the necessary consideration at the organizational level. Five items were employed in this study to measure consideration.

Likewise, it was assumed that the information system artifact is a multi-dimensional construct in the context of this study. According to Erol et al. [25] and Venkatesh [35], they presented the necessary imputes for the evaluation of the ISA within the organization. A total of 16 items were employed to estimate the ISA followed by organizational resilience being conceptualized with the definition afforded by Wing and Wai [23]. In this case, organizational resilience was associated with the response to a disaster, adjusting to adapt and revert to normality following the adversity, having a plan B in accommodating the need to work with the changing conditions. Finally, organizational resilience was evaluated using six statements. Complete instrument presented in Appendix A.

\subsection{Common Method Variance (CMV)}

In social science research, using a questionnaire is acknowledged as a standard method to collect data and perform the analysis. This approach leads to a standard method to determine the variance and the measurement of error [39]. As the researcher constructed a common variance with other related constructs within this study, the treatment of the common method variance (CMV) was made at the pre-data-collection stage, at the data collection stage as well as the analysis stage. Therefore, a common scale was adopted in this study and Harman's [40] single-factor analysis was used to estimate the CMV. The guidelines suggested that the single factor must be extracted if there is less than a $40 \%$ variance in order to establish the controlled level of CMV within the study's constructs [39]. For this study, the results of the Harman single-factors that were extracted accounted for less than $40 \%$ [40]. Therefore, CMV was not a serious issue for this study. 


\subsection{Multivariate Normality}

The multivariate normality was not considered to be a critical issue for the SEM-PLS given this technique is a non-parametric one. Nevertheless, the suggestion of Peng and Lai [41] was adopted in testing the multivariate normality of the data in order to justify the use of SEM-PLS with the non-normal data set. The online tool, 'web power', was used to test the multivariate normality of the data set which provided the results with skewness and kurtosis coefficients with the $p$-value for the data set. The data were considered to be non-normal if the $p$-value for the Mardia's multivariate coefficient was less than 0.05 [42]. The results of our data set suggest that the data were non-normal.

\subsection{Data Analysis Method}

Partial least square modeling (PLS-SEM) is a popular analytical technique in working with latent variables in investigating the causal effect of endogenous variables. PLS-SEM was used in this study given the nature of the study was explorative in design, and the data with non-normality [43]. The recommendation presented by Hair et al. [43] in reporting the results was also adopted in this study. However, the issue of endogeneity was recently discussed in PLS-SEM given it utilized the combination of principal components analysis with regression-based path analysis. The two-stage approach in PLS-SEM estimated the path coefficients between the latent variables and their indicators at the measurement model estimation and between the latent variables at the structural model stage [43]. PLS-SEM was employed for predictive or explanatory modeling purposes where the aim was to predict future managerial actions rather than interpreting the structural paths. Although, controlling for endogeneity is not advisable in predictive modeling whereas for explanatory modeling, controlling for the endogeneity is essential. Moreover, as PLS-SEM adopts the predictive-explanatory approach, the researcher needed to decide on the consideration of the endogeneity issue. The recommended indicator loading employed was 0.70 , given the acceptable level for explorative studies is 0.40 for each indicator. Cronbach's alpha $(\alpha)$ and composite reliability $(\mathrm{CR})$ were employed to report on the internal consistency of the constructs. The recommended score based on the literature for $(\alpha)$ and $(\mathrm{CR})$ is 0.70 or higher [38]. However, (CR) is considered a better indicator of internal reliability compared to $(\alpha)$.

Additionally, the average variance extracted (AVE) value must be 0.50 or higher for each construct thereby indicating more than half of the variance of the construct explained by the construct indicators. Here, the variance inflation factor (VIF) represents the inflation of variance given the presence of the multicollinearity within the study constructs. The VIF of less than 3.3 depicts that the construct has an acceptable VIF [43]. The discriminant validity in PLS-SEM was verified with the cross-loading, Fornell-Larcker, and heterotrait-monotrait ratio (HTMT). The cross-loading table, in this case, helped to identify that the loadings of the items must be more for its own construct rather than for the other constructs. Here, the Fornell-Larcker criterion needed to be higher than 0.70 for its own construct to provide evidence of discriminate validity (DV). Although, in this case, a new test of HTMT is recommended for DV as the values must be less than 0.90 [38].

The measurement model was represented with the $r^{2}$ that denotes the explanation power of the endogenous variables with the exogenous variables. The effect size $\left(f^{2}\right)$ and $Q^{2}$ are the estimates of the measurement model. The effect size $\left(f^{2}\right)$ signifies the effect of each exogenous variable on the endogenous variable. Cohen's [44] offers the guidelines to the means of $\left(f^{2}\right)$. The effect size of 0.30 , 0.15 and 0.02 presents the large, medium, and small effects respectively. The $Q^{2}$ is the measure of the predictive relevance for the model in terms of the accuracy of the input variables in predicting the outcome variables. The $Q^{2}$ value of $0.02,0.15$, and 0.35 indicates the small, medium, and large predictive relevancy of the model, respectively [43]. 


\section{Data Analysis}

\subsection{Testing Endogeniety}

Notwithstanding, given the issue of endogeneity is critical in employing regression-based methods like PLS-SEM [45], the first test conducted in this study was for non-normality of the independent variables. The normality test revealed that the input variables were non-normal (see Section 3.4).

The Gaussian copula approach was next undertaken as suggested by Park and Gupta [46]. The method is based on the inclusion of error-term in the structural model to test the effect of exogenous variables' errors toward the exogenous variables [45]. The results of the Gaussian copulas (i.e., commitment, community, communication, connection, competency, coordination and consideration) for all input variables were found to be insignificant at ( $p>0.05)$, as depicted in Table 1 . As a result, we conclude that endogeneity is not a critical issue for this study and does not impact the robustness of the structural model that generated the results of this study.

Table 1. Endogeniety test by use of the Gaussian copula approach.

\begin{tabular}{lllll}
\hline Test & Path & Coefficient & T-Value & P-Value \\
\hline Gaussian copula (endogenous & COM $\rightarrow$ CMM & 0.265 & 2.532 & 0.012 \\
variable: CMM, & CMP $\rightarrow$ COM & 0.425 & 5.682 & 0.000 \\
COM, CIT, CMP, CON, COD, CNS) & CMM $\rightarrow$ CIT & 0.037 & 0.720 & 0.472 \\
& CON $\rightarrow$ CMP & 0.363 & 3.014 & 0.003 \\
& CMM $\rightarrow$ CON & 0.417 & 2.927 & 0.004 \\
& CON $\rightarrow$ COD & 0.045 & 0.231 & 0.817 \\
& CMM $\rightarrow$ CNS & 0.225 & 1.177 & 0.240 \\
& CMMc $\rightarrow$ CMM & 0.219 & 0.514 & 0.608 \\
& COMc $\rightarrow$ COM & -0.056 & 0.003 & 0.998 \\
& CITc $\rightarrow$ CIT & -0.002 & 0.028 & 0.978 \\
& CMPc $\rightarrow$ CMP & -0.013 & 0.005 & 0.996 \\
CONc $\rightarrow$ CON & 0.173 & 0.109 & 0.913 \\
CODc $\rightarrow$ COD & 0.326 & 1.004 & 0.316 \\
& CNSc $\rightarrow$ CNS & -0.005 & 1.763 & 0.078 \\
\hline
\end{tabular}

Note: CMM: commitment; COM: communication; CIT: community; CMP: competency; CON: connection; COD: coordination; CNS: consideration. $\mathrm{c}$ indicates the copula term in the model.

\subsection{Descriptive Statistics}

The sample size for the final analysis was 252 as mentioned earlier based on the number of responses received from participants. The response rate was just above $50 \%$ of the total number of questionnaires distributed. The final data set based on gender used for the analysis comprised of females (43.7\%) with the remainder being males. The vast majority of respondents were associated with the elderly age group, 30 years or older. The respondents with ages less than 30 years represented $24.2 \%$ with the largest segment of the sample being unmarried representing $(68 \%)$.

As noted in Table 2, the education level of the sample consisted mainly of respondents holding a college degree or above, with only $24 \%$ of respondents with an educational level less than a college degree. Therefore, the respondents in this study were classified as being highly educated and suitable to participate in this study. Regarding the experience of respondents, $71 \%$ had 6 years or more experience indicating that the skills and experience of the sample relating to the perceived phenomenon of resilience as well as the ISA in this very study were appropriate. Notably, experiences can provide valuable insights in assessing the collective inventory of perceptions and its influence on organizational resilience. The population of the sample consisted mostly of Malaysian nationals (91.2\%). 


\subsection{Validity and Reliability}

As described earlier, the endorsement recommended in the study by Hair et al. (2014) was adopted where Cronbach's alpha $(\alpha)$ and composite reliability (CR) must be 0.70 or higher for each construct, as shown in Table 3. Here, $(\alpha)$ and $(\mathrm{CR})$ for each construct are shown to be 0.803 and 0.874 , respectively. The Cronbach's alpha reports the inter-correlational estimate of the question items for each construct showing that 0.803 is the minimum value with the other constructs having Cronbach's alpha values above the prescribed limit of 0.70 . Therefore, the Cronbach's alpha and CR values denote that the constructs are reliable. The (AVE) for all items in each construct also needs to be higher than 0.50 score in order to establish the convergent validity $(\mathrm{CV})$ to confirm the unidimensionality. The items in the table show that $\mathrm{CV}$ is satisfactory.

Table 2. Profile of the Respondents and SMEs.

\begin{tabular}{lcclcc}
\hline & $\mathbf{n}$ & $\mathbf{\%}$ & & $\mathbf{n}$ & $\%$ \\
\hline Gender & & & Age & & \\
Male & 142 & $56.3 \%$ & Less than 30 years of age & 61 & $24.2 \%$ \\
Female & 110 & $43.7 \%$ & 30-39 years of age & 90 & $35.7 \%$ \\
Total & 252 & $100 \%$ & $40-49$ years of age & 76 & $30.1 \%$ \\
& & & 50-59 years of age & 25 & $9.92 \%$ \\
Education & & & 60 years of age or above & 0 & $0 \%$ \\
SPM & 17 & $6.7 \%$ & Total & 252 & $100 \%$ \\
Diploma & 45 & $17.9 \%$ & & & \\
Degree & 164 & $65.1 \%$ & Marital Status & 173 & $68.6 \%$ \\
Master & 22 & $8.7 \%$ & Single & 73 & $28.9 \%$ \\
Others & 4 & $1.6 \%$ & Married & 3 & $1.19 \%$ \\
Total & 252 & $100 \%$ & Divorced & 0 & $0 \%$ \\
& & & Separated & 3 & $1.19 \%$ \\
Working Experience & & & Widowed & 252 & $100 \%$ \\
1-5 Years & 72 & $28.5 \%$ & Total & & \\
5-10 Years & 95 & $37.6 \%$ & & 230 & $91.2 \%$ \\
10-15 Years & 32 & $12.6 \%$ & Nationality & 22 & $8.7 \%$ \\
15-20 Years & 16 & $6.3 \%$ & Malaysian & 252 & $100 \%$ \\
20-25 Years & 27 & $10.7 \%$ & Non-Malaysian & & \\
Over 25 Years & 10 & $3.9 \%$ & Total & & \\
Total & 252 & $100 \%$ & & & \\
\hline
\end{tabular}

Table 3. Reliability analysis.

\begin{tabular}{lccccc}
\hline \multicolumn{1}{c}{ Variables } & Number of Items & Cronbach's Alpha & Composite Reliability & AVE & VIF \\
\hline Commitment & 5 & 0.916 & 0.937 & 0.750 & 1.799 \\
Communication & 5 & 0.839 & 0.886 & 0.610 & 2.012 \\
Community & 5 & 0.852 & 0.894 & 0.628 & 2.237 \\
Competency & 5 & 0.803 & 0.864 & 0.560 & 2.132 \\
Connection & 5 & 0.854 & 0.895 & 0.631 \\
Coordination & 4 & 0.892 & 0.919 & 0.697 \\
Consideration & 5 & 0.854 & 0.899 & 0.691 \\
Information System Artifact & 16 & 0.945 & 0.951 & 1.619 \\
Organizational Resilience & 6 & 0.826 & 0.874 & 0.551 & - \\
\hline
\end{tabular}

Note: CMM: commitment; COM: communication; CIT: community; CMP: competency; CON: connection; COD: coordination; CNS: consideration; ISA: information system artifact; ORE: organizational resilience; AVE: average variance extracted.

To test the DV the loading for each item and cross-loading needed to be checked in which the results showed that the item loads on their respective variables fulfilled the assumption of DV (refer Table 4). A further test for DV was also needed to check against the Fornell-Larcker criterion in which the results were found to be satisfactory, and the values were in an acceptable range. Another suggested test for DV was the HTMT ratio where the values must be 0.90 or less in proving that the study has DV. The results depicted in Table 4 show that the study has no evidence regarding the lack of DV. 
Table 4. Outer loading and cross loadings.

\begin{tabular}{|c|c|c|c|c|c|c|c|c|c|}
\hline & CMM & COM & CIT & CMP & $\mathrm{CON}$ & COD & CNS & ISA & ORE \\
\hline Commitment. Item-1 & 0.866 & 0.325 & 0.388 & 0.290 & 0.183 & 0.270 & 0.386 & 0.435 & 0.344 \\
\hline Commitment. Item-2 & 0.827 & 0.383 & 0.405 & 0.265 & 0.111 & 0.145 & 0.346 & 0.490 & 0.359 \\
\hline Commitment. Item-3 & 0.865 & 0.339 & 0.421 & 0.284 & 0.141 & 0.131 & 0.332 & 0.418 & 0.346 \\
\hline Commitment. Item-4 & 0.877 & 0.446 & 0.452 & 0.260 & 0.148 & 0.139 & 0.256 & 0.390 & 0.400 \\
\hline Commitment. Item-5 & 0.893 & 0.350 & 0.412 & 0.207 & 0.125 & 0.053 & 0.289 & 0.519 & 0.289 \\
\hline Communication. Item-1 & 0.406 & 0.673 & 0.390 & 0.168 & 0.084 & 0.139 & 0.427 & 0.435 & 0.325 \\
\hline Communication. Item-2 & 0.213 & 0.822 & 0.484 & 0.183 & 0.044 & 0.212 & 0.198 & 0.439 & 0.431 \\
\hline Communication. Item-3 & 0.284 & 0.848 & 0.398 & 0.309 & 0.023 & 0.083 & 0.234 & 0.421 & 0.352 \\
\hline Communication. Item -4 & 0.346 & 0.765 & 0.458 & 0.298 & 0.035 & -0.055 & 0.241 & 0.429 & 0.246 \\
\hline Communication. Item -5 & 0.290 & 0.787 & 0.308 & 0.347 & 0.130 & 0.114 & 0.123 & 0.550 & 0.317 \\
\hline Community. Item-1 & 0.411 & 0.319 & 0.772 & 0.228 & 0.094 & 0.138 & 0.218 & 0.281 & 0.337 \\
\hline Community. Item-2 & 0.384 & 0.472 & 0.817 & 0.275 & 0.024 & 0.054 & 0.243 & 0.375 & 0.344 \\
\hline Community. Item-3 & 0.269 & 0.378 & 0.776 & 0.230 & -0.013 & -0.056 & 0.183 & 0.388 & 0.359 \\
\hline Community. Item-4 & 0.171 & 0.497 & 0.763 & 0.279 & 0.068 & -0.043 & 0.250 & 0.347 & 0.346 \\
\hline Community. Item-5 & 0.468 & 0.464 & 0.832 & 0.308 & 0.085 & 0.100 & 0.235 & 0.377 & 0.400 \\
\hline Competency. Item-1 & 0.398 & 0.456 & 0.319 & 0.756 & 0.216 & 0.381 & 0.182 & 0.459 & 0.289 \\
\hline Competency. Item-2 & 0.386 & 0.540 & 0.375 & 0.830 & 0.160 & 0.420 & 0.156 & 0.388 & 0.334 \\
\hline Competency. Item-3 & 0.254 & 0.469 & 0.352 & 0.701 & 0.153 & 0.438 & 0.195 & 0.428 & 0.330 \\
\hline Competency. Item-4 & 0.275 & 0.445 & 0.312 & 0.697 & 0.039 & 0.459 & 0.176 & 0.439 & 0.368 \\
\hline Competency. Item -5 & 0.371 & 0.533 & 0.313 & 0.749 & 0.108 & 0.433 & 0.147 & 0.436 & 0.344 \\
\hline Connection. Item-1 & 0.673 & 0.108 & 0.548 & 0.439 & 0.827 & 0.040 & 0.128 & 0.263 & 0.343 \\
\hline Connection. Item-2 & 0.406 & 0.229 & 0.560 & 0.460 & 0.786 & -0.026 & 0.009 & 0.237 & 0.223 \\
\hline Connection. Item-3 & 0.213 & 0.253 & 0.535 & 0.559 & 0.843 & 0.077 & 0.071 & 0.281 & 0.229 \\
\hline Connection. Item-4 & 0.284 & 0.226 & 0.524 & 0.252 & 0.741 & -0.007 & 0.002 & 0.216 & 0.288 \\
\hline Connection. Item-5 & 0.346 & 0.219 & 0.512 & 0.435 & 0.770 & 0.035 & -0.051 & 0.281 & 0.162 \\
\hline Coordination. Item-1 & 0.290 & 0.319 & 0.274 & 0.393 & 0.270 & 0.814 & -0.055 & 0.129 & 0.334 \\
\hline Coordination. Item -2 & 0.411 & 0.472 & 0.268 & 0.419 & 0.145 & 0.791 & 0.386 & 0.135 & 0.330 \\
\hline Coordination. Item-3 & 0.384 & 0.378 & 0.255 & 0.309 & 0.131 & 0.840 & 0.346 & 0.078 & 0.368 \\
\hline Coordination. Item-4 & 0.269 & 0.497 & 0.227 & 0.518 & 0.139 & 0.877 & 0.332 & 0.103 & 0.344 \\
\hline Coordination. Item -5 & 0.171 & 0.464 & 0.302 & 0.470 & 0.053 & 0.814 & 0.256 & 0.086 & 0.343 \\
\hline Consideration. Item-1 & 0.468 & 0.261 & 0.055 & 0.525 & 0.139 & 0.057 & 0.894 & 0.389 & 0.223 \\
\hline Consideration. Item-2 & 0.398 & 0.319 & 0.128 & 0.455 & 0.212 & 0.035 & 0.711 & 0.222 & 0.229 \\
\hline Consideration. Item-3 & 0.386 & 0.221 & 0.032 & 0.498 & 0.083 & 0.024 & 0.843 & 0.435 & 0.288 \\
\hline Consideration. Item-4 & 0.254 & 0.253 & 0.026 & 0.304 & -0.055 & 0.044 & 0.859 & 0.490 & 0.162 \\
\hline Consideration. Item -5 & 0.275 & 0.313 & 0.001 & 0.278 & 0.114 & 0.000 & 0.854 & 0.418 & 0.334 \\
\hline I.S. Artifact. Item-1 & 0.371 & 0.062 & 0.282 & 0.228 & 0.138 & -0.025 & 0.057 & 0.721 & 0.330 \\
\hline I.S. Artifact. Item-2 & 0.673 & 0.113 & 0.108 & 0.237 & 0.054 & 0.059 & 0.035 & 0.761 & 0.368 \\
\hline I.S. Artifact. Item-3 & 0.406 & 0.036 & 0.012 & 0.331 & -0.056 & -0.011 & 0.024 & 0.736 & 0.344 \\
\hline I.S. Artifact. Item-4 & 0.213 & 0.031 & -0.027 & 0.082 & -0.043 & -0.006 & 0.044 & 0.766 & 0.343 \\
\hline I.S. Artifact. Item-5 & 0.284 & 0.016 & 0.045 & 0.167 & 0.100 & 0.047 & 0.000 & 0.673 & 0.223 \\
\hline I.S. Artifact. Item-6 & 0.346 & 0.163 & 0.339 & 0.018 & 0.381 & 0.018 & -0.025 & 0.724 & 0.229 \\
\hline I.S. Artifact. Item-7 & 0.263 & 0.067 & 0.169 & 0.040 & 0.420 & 0.046 & 0.059 & 0.790 & 0.288 \\
\hline I.S. Artifact. Item-8 & 0.213 & 0.015 & 0.445 & -0.001 & 0.438 & 0.059 & -0.011 & 0.751 & 0.162 \\
\hline I.S. Artifact. Item-9 & 0.257 & 0.009 & 0.485 & 0.263 & 0.459 & 0.114 & -0.006 & 0.741 & 0.334 \\
\hline I.S. Artifact. Item-10 & 0.214 & -0.009 & 0.442 & 0.167 & 0.433 & 0.163 & 0.047 & 0.739 & 0.330 \\
\hline I.S. Artifact. Item-11 & 0.221 & 0.189 & 0.465 & 0.058 & 0.040 & 0.149 & 0.018 & 0.739 & 0.368 \\
\hline I.S. Artifact. Item-12 & 0.092 & 0.101 & 0.439 & 0.109 & -0.026 & 0.150 & 0.046 & 0.785 & 0.344 \\
\hline I.S. Artifact. Item-13 & 0.100 & 0.345 & 0.434 & 0.103 & 0.077 & 0.152 & 0.059 & 0.791 & 0.343 \\
\hline I.S. Artifact. Item-14 & 0.070 & 0.409 & 0.490 & 0.258 & -0.007 & -0.034 & 0.114 & 0.701 & 0.223 \\
\hline I.S. Artifact. Item-15 & 0.002 & 0.261 & 0.421 & 0.189 & 0.035 & -0.034 & 0.163 & 0.742 & 0.229 \\
\hline I.S. Artifact. Item-16 & 0.263 & 0.319 & 0.371 & 0.374 & -0.188 & -0.017 & 0.149 & 0.706 & 0.288 \\
\hline O. Resilience. Item-1 & 0.213 & 0.221 & 0.369 & 0.437 & -0.115 & 0.051 & 0.150 & 0.295 & 0.734 \\
\hline O. Resilience. Item-2 & 0.257 & 0.253 & 0.422 & 0.445 & -0.159 & 0.015 & 0.152 & 0.212 & 0.780 \\
\hline O. Resilience. Item-3 & 0.214 & 0.313 & 0.324 & 0.402 & -0.212 & 0.042 & -0.034 & 0.197 & 0.688 \\
\hline O. Resilience. Item-4 & 0.221 & 0.062 & 0.376 & 0.420 & -0.180 & 0.034 & -0.034 & 0.148 & 0.744 \\
\hline O. Resilience. Item-5 & 0.092 & 0.113 & 0.353 & 0.447 & -0.058 & 0.000 & -0.017 & 0.145 & 0.763 \\
\hline O. Resilience. Item-6 & 0.100 & 0.036 & 0.442 & 0.520 & -0.004 & -0.015 & 0.057 & 0.115 & 0.678 \\
\hline \multicolumn{10}{|c|}{ Fronell-Larcker Criterion } \\
\hline Commitment & 0.866 & & & & & & & & \\
\hline Communication & 0.270 & 0.781 & & & & & & & \\
\hline Community & 0.618 & 0.427 & 0.792 & & & & & & \\
\hline Competency & 0.570 & 0.467 & 0.655 & 0.748 & & & & & \\
\hline Connection & 0.343 & 0.087 & 0.335 & 0.348 & 0.794 & & & & \\
\hline Coordination & 0.070 & 0.169 & 0.068 & 0.088 & 0.425 & 0.835 & & & \\
\hline Consideration & 0.029 & 0.545 & 0.054 & 0.140 & -0.214 & 0.030 & 0.831 & & \\
\hline Information System Artifact & 0.498 & 0.456 & 0.579 & 0.607 & 0.324 & 0.134 & 0.215 & 0.742 & \\
\hline Organizational Resilience & 0.254 & 0.261 & 0.422 & 0.351 & 0.201 & 0.002 & 0.197 & 0.463 & 0.732 \\
\hline \multicolumn{10}{|c|}{ Heterotrait-Monotrait Ratios } \\
\hline Commitment & - & & & & & & & & \\
\hline Communication & 0.302 & - & & & & & & & \\
\hline Community & 0.699 & 0.504 & - & & & & & & \\
\hline Competency & 0.664 & 0.551 & 0.778 & - & & & & & \\
\hline Connection & 0.390 & 0.118 & 0.389 & 0.420 & - & & & & \\
\hline Coordination & 0.070 & 0.208 & 0.092 & 0.105 & 0.483 & - & & & \\
\hline Consideration & 0.057 & 0.652 & 0.150 & 0.219 & 0.259 & 0.089 & - & & \\
\hline Information System Artifact & 0.534 & 0.507 & 0.638 & 0.689 & 0.357 & 0.152 & 0.228 & - & \\
\hline Organizational Resilience & 0.289 & 0.308 & 0.493 & 0.422 & 0.240 & 0.091 & 0.235 & 0.518 & - \\
\hline
\end{tabular}

Note: CMM: commitment; COM: communication; CIT: community; CMP: competency; CON: connection; COD: coordination; CNS: consideration; ISA: information system artifact; ORE: organizational resilience. 


\subsection{Path Analysis}

Given the study performed structural model analysis in PLS-SEM to achieve reliability and validity of the study constructs, the next stage of analysis involved the measurement model. The adjusted $r^{2}$ value for the seven input variables (i.e., commitment, communication, community, competency, connection, coordination, consideration) on the ISA indicated that $46.5 \%$ of the variance in the ISA could be explained using these input variables. The predictive relevance $\left(\mathrm{Q}^{2}\right)$ value for the part of the model was 0.228 indicating a medium predictive relevance. The adjusted $r^{2}$ value for the ISA on the organizational resilience was $21.1 \%$, indicating that the ISA can explain the $21.1 \%$ variance in organizational resilience. The predictive relevance $\left(\mathrm{Q}^{2}\right)$ value for this component of the model was 0.098 thereby indicating a small predictive relevance.

The standardized path coefficients, $t$-values and significance level are displayed in Table 5 showing that the path coefficient for organizational commitment to organizational resilience is $(\beta=0.126$, $p=0.006)$, which therefore supports $\mathrm{H} 1$. The result also indicates that the effect of organizational commitment on ISA is positive and significant. The path coefficient for organizational communication to organizational resilience is $(\beta=0.120, p=0.011)$, indicating a positive and significant effect of organizational communication on the ISA. The result demonstrates that $\mathrm{H} 2$ is supported. The path coefficient for the organizational perception of community on the ISA is $(\beta=0.223, p=0.000)$ indicating that the effect of community perception on the ISA is positive and significant, thereby supporting H3. The path coefficient for organizational competency on the ISA is $(\beta=0.272, p=0.000)$, thereby indicating a positive effect of the competency on the ISA and offering evidence to support H4. The path coefficient for connection to the ISA is $(\beta=0.123, p=0.035)$, therefore indicating that the effect of organizational connection perception on the ISA is positive and significant, thereby supporting $\mathrm{H} 5$. The path coefficient for coordination to the ISA is $(\beta=0.122, p=0.007)$, indicating that effect of organizational coordination on the ISA is positive and significant, providing evidence in supporting H6. The path coefficient for consideration to the ISA is $(\beta=0.010, p=0.436)$, suggesting that the effect of organizational consideration on the ISA is negative and insignificant providing evidence not supporting H7. The path coefficient for the ISA to organizational resilience is $(\beta=0.463, p=0.008)$, showing that the effect of the ISA on organizational resilience is positive and significant therefore providing sufficient evidence to support H8. The results of the path coefficients are displayed in Table 5.

Table 5. Hypothesis testing.

\begin{tabular}{lccccccc}
\hline & Hypothesis & Coefficient & t-Values & Sig. & $\boldsymbol{r}^{\mathbf{2}}$ & $f^{\mathbf{2}}$ & Decision \\
\hline H1 & CMM $\rightarrow$ ISA & 0.126 & 2.512 & 0.006 & & 0.017 & Supported \\
H2 & COM $\rightarrow$ ISA & 0.120 & 2.295 & 0.011 & & 0.014 & Supported \\
H3 & CIT $\rightarrow$ ISA & 0.223 & 3.635 & 0.000 & & 0.043 & Supported \\
H4 & CMP $\rightarrow$ ISA & 0.272 & 3.782 & 0.000 & 0.465 & 0.067 & Supported \\
H5 & CON $\rightarrow$ ISA & 0.123 & 1.818 & 0.035 & & 0.018 & Supported \\
H6 & COD $\rightarrow$ ISA & 0.122 & 2.472 & 0.007 & & 0.018 & Supported \\
H7 & CNS $\rightarrow$ ISA & 0.010 & 0.162 & 0.436 & & 0.000 & Not supported \\
H8 & ISA $\rightarrow$ ORE & 0.463 & 6.387 & 0.008 & 0.211 & 0.273 & Supported \\
\hline
\end{tabular}

Note: CMM: commitment; COM: communication; CIT: community; CMP: competency; CON: connection; COD: coordination; CNS: consideration; ISA: information system artifact; ORE: organizational resilience.

\subsection{Mediating Effects}

The ISA meditational effect was tested in order to determine organizational, communication, community, competency, connection, coordination, and consideration on organizational resilience. The findings of the meditational effect of the ISA are displayed in Table 5. The results indicate that the perception of commitment in the MDEC registered enterprises is mediated by the ISA for organizational resilience ( $\beta=0.058, p=0.016)$. The outcome for the perception of communication in the MDEC registered enterprises is mediated by the ISA for organizational resilience $(\beta=0.056$, 
$p=0.016)$. Accordingly, the results demonstrate that the perception of community in the MDEC registered enterprises is mediated by the ISA for organizational resilience $(\beta=0.103, p=0.002)$.

The results in Table 6 indicate that the perception of competency in the MDEC registered enterprises is mediated by the ISA for organizational resilience $(\beta=0.126, p=0.000)$. The results also demonstrate that the perception of connection in the MDEC registered enterprises is mediated by the ISA for organizational resilience $(\beta=0.057, p=0.039)$. Furthermore, the results also depict that the perception of connection in the MDEC registered enterprises is mediated by the ISA for organizational resilience ( $\beta=0.056, p=0.018)$ and the results of the perception of consideration in the MDEC registered enterprises are not mediated by the ISA for organizational resilience $(\beta=0.057, p=0.436)$.

Table 6. Meditating effect.

\begin{tabular}{llllll}
\hline & $\beta$ & CI-min & CI-max & Sig. & Decision \\
\hline $\mathrm{CMM} \rightarrow$ ISA $\rightarrow$ ORE & 0.058 & 0.016 & 0.106 & 0.016 & Mediation \\
$\mathrm{COM} \rightarrow$ ISA $\rightarrow$ ORE & 0.056 & 0.014 & 0.099 & 0.016 & Mediation \\
$\mathrm{CIT} \rightarrow$ ISA $\rightarrow$ ORE & 0.103 & 0.044 & 0.162 & 0.002 & Mediation \\
$\mathrm{CMP} \rightarrow$ ISA $\rightarrow$ ORE & 0.126 & 0.067 & 0.191 & 0.000 & Mediation \\
$\mathrm{CON} \rightarrow$ ISA $\rightarrow$ ORE & 0.057 & 0.007 & 0.011 & 0.039 & Mediation \\
$\mathrm{COD} \rightarrow$ ISA $\rightarrow$ ORE & 0.056 & 0.016 & 0.105 & 0.018 & Mediation \\
$\mathrm{CNS} \rightarrow$ ISA $\rightarrow$ ORE & 0.005 & -0.038 & 0.060 & 0.436 & No Mediation
\end{tabular}

Note: CMM: commitment; COM: communication; CIT: community; CMP: competency; CON: connection; COD: coordination; CNS: consideration; ISA: information system artifact; ORE: organizational resilience.

\section{Discussion}

SMEs are acknowledged as forming an integral part of every economy globally and are similar in terms of their operations and methods in producing goods and in delivering services. Although this is not quite correct as not all SMEs are similar but are different based on their collective stock of behavioral capacities which are stored in the shape and form of their employees and how they use the ISA. This, in fact, becomes the source to transcend and transition employees' capacities into shaping the resilience of an SME [3].

In this study, the collective stock of employee behavior was found to have a positive effect on the ISA, and that the ISA functions as a mediator amid the collective capacities of employees and organizational resilience. Seven collective capacities were identified as mentioned earlier relative to employees' namely collective commitment, communication, community, competency, connection, consideration, and organizational coordination [7]. Hypotheses were formulated, hypothesizing that these collective employee capacities not only have a positive effect on the ISA utilized by SMEs but also that the ISA has a positive effect on organizational resilience among SMEs registered with the MDEC in Malaysia. Additionally, the hypotheses presumed that the ISA mediates the relationship between these seven collective capacities of organizational employees and organizational resilience in SMEs registered with the MDEC in Malaysia.

The first hypothesis assessed the effect of collective commitment perception that prevails in the enterprise in light of ISA, as the significantly positive outcomes signify that enterprise-level commitment has an impact on ISA. Collective commitment positively indicates the application of ISA across SMEs supported by a dedicated and well-skilled workforce in effectively implementing transformational change across the enterprise. For many employees, the ISA is challenging, but with the broad commitment of the enterprise, the implementation of the ISA will occur efficiently. The second hypothesis indicated the significantly positive effect of communication on ISA. Similar results were reported by previous studies [6]. Effective communication amidst an enterprise positively influences the application of ISAs across SMEs. Therefore, collective communication has a positive influence on the ISAs. Given a reliable and effective communication system exists among SME employees, embedding the ISA into the enterprise is reasonably easy to achieve. Also, implementation issues are quickly resolved given good communication exists across all levels of the organization, (i.e., among 
employees and between management and employees, etc.). The third hypothesis measured the effect of the collective community perception prevailing in the enterprise on the enterprise ISA where the findings indicated that collective community perception has a positive and significant effect on the enterprise ISA [30]. Accordingly, this suggests that the collective perception of the community has a positive effect on the enterprise ISA. The collective perception of the community makes it significantly easier in accepting ISAs given that the communication network already exists and is effective in helping employees work collaboratively on the newly established ISA [8]. The fourth hypothesis assessed the effect of the collective competency perception existing in the enterprise on the enterprise ISA where the findings indicated that collective community perception has a positive and significant effect on enterprise ISA. Accordingly, this indicates that the collective perception of competency has a positive effect on enterprise ISA. The collective perception of competency facilitates the implementation of the ISA as skills and competencies are needed to work and integrate efficiently with the newly implemented ISA [8].

The fifth hypothesis appraised the effect of the collective connection perception that subsists in the enterprise on enterprise ISA where the findings showed that collective connection perception has a positive and significant effect on enterprise ISA. The collective connection is the concept of bonding amongst SME employees. Bonding helps employees work collaboratively together as a collective workforce having a common purpose, mindset, and attitude [5]. In this case, the collective connection positively affects the ISA. The sixth hypothesis was formulated to calculate the effect of the collective coordination perception that occurs in the enterprise on enterprise ISA where the findings indicated that the collective coordination perception has a positive and significant effect on enterprise ISA. The collective sense of coordination amongst employees facilitates in establishing new innovative systems like the ISAs. The perception of coordination is similar to the notion of helping each other in difficult or challenging situations. In this case, the ISA is positively impacted by the collective perceived sense of coordination [5]. The seventh hypothesis was to calculate the effect of the collective consideration perception in the enterprise on the enterprise ISA where the findings indicated that collective consideration has a positive and significant effect on enterprise ISA. Collective consideration makes it significantly easier to implement ISAs, given that employees collectively (as one body) perceive their importance to the enterprise and themselves as employees. Accordingly, these findings are in agreement with the study conducted by Al-Natour and Benbasat [37]. The effects of collective behavioral capacities across SMEs positively and significantly influenced the perception of ISAs in SMEs. Hence, management of behavioral capacities is crucial to arrive at positive effects on the perception of ISAs.

Hypothesis 9 were formulated to estimate the mediation of the ISA between the collective perception of commitment, communication, connection, coordination, consideration, community, competency, and organizational resilience. The findings from the mediation analysis confirmed that the perception of ISA mediates the relationship between commitment, communication, connection, coordination, community, competency, and organizational resilience (see Table 6). The findings of this study also established that employees' collective perception (i.e., commitment, communication, connection, coordination, community, and competency) act through the ISA in helping the organization to become more resilient [37]. However, the result of the ISA mediation between collective consideration and organizational resilience was not established which indicates that the employees are unsure about the objectivity of the ISA for the resilience of the organization. Hypothesis 8 was formulated to measure the effect of the ISA on enterprise resilience among the SMEs registered with MDEC Malaysia. The finding supports the claim that ISA implementation in SMEs has a positive and significant effect on enterprise resilience.

\section{Conclusions}

This study investigated and extended the work of [3,34] in testing and extending the role of the ISA for organization resilience. Using the ISA as the mediator for the collective organizational capacities 
for organizational resilience $[25,29]$, the connection was observed for the organizational collective perception with the ISA for the organizational resilience. The findings of the study also confirmed that the collective perception of the organizational employees towards organizational commitment, communication, community, competency, connection, and coordination have a significant effect on the organizational resilience through the ISA [37]. Accordingly, this study demonstrated that employee's perception as a resource positively enhances the use for the ISA in which the ISA affects organizational resilience [30].

In this case, the employees' perception of commitment positively affects the ISA as community-wide acceptance of ISA enables the shared belongings and ownership of work, therefore, affecting organizational resilience. Additionally, the perception of communication at the SME level makes it relatively easier for employees to openly communicate with fellow employees and management. Accordingly, the elevated perception of communication amongst SME employees will impact the ISA, in which will influence organization resilience. The employees' perception of community was also found to affect ISAs as the community-wide acceptance of ISAs enables employees to work collaboratively on common work routines and practices within the firm, in turn, impacting organizational resilience positively.

Moreover, the perception of competency at the SME level helps to turn around what was initially perceived to be impossible into something that is achievable. As such, the higher perception of competency among SME employees impacts the ISA, in which influences organizational resilience. Similarly, the collective perceptions of the connection amongst SME employees acts as a binding force among employees and facilitate the implementation of ISA thereby leading to organizational resilience. Likewise, collective coordination helps to implement and perform new and innovative practices like ISA which positively influences the ISA and organizational resilience. However, collective perceived consideration does not impact the ISA and has no effect on organizational resilience. Therefore, the study contributes to the theory and practice in this field by employing the collective perception framework using seven factors shared by the contradictory understandings of organizational resilience and builds upon the ISA as a convergent point in order to achieve organizational resilience.

This study contributes to a substantial number of prevailing struggles revealed in the literature by discovering and augmenting the insights concerning organizational resilience [3] as a process that channels through ISA in the form of collective stock of behavioral organizational capacities $[10,37]$. The study outcomes offer practical understanding for managers and policymakers regarding the fact that the most critical resource within an organization is its people, and the combination of ISA with behavioral capacities of employees enables an organization to become more resilient [1]. Inevitably, this paves the path for any organization to achieve resilience by implementing ISA and by correcting the collective perception of employees in achieving resilience at the organizational level. The management in SMEs need to harness communication, consideration, and establishment of the community's perception toward SME employees, which can lead to better commitment, coordination, connection, and competency to perform for the SMEs, along with the application of ISAs. ISAs facilitate working and realize the impact of communication, connection, and consideration. The implementation of ISA helps to have common organizational consideration. Thus, information about ISA is made available to all employees to facilitate work based on competency building, along with the notion of community, where everyone works in coordination with having higher commitment for the achievement of organizational resilience. Indeed, the policymaker also needs to establish the required standard and policy in that the ISA must form part of the SME in achieving better resilience for the organization. Policy guidelines facilitate SMEs in enabling ISA implementation to achieve resilience amongst SMEs. As such, the implantation of the ISA will help to provide the necessary condition to achieve a resilient organization at the local, state, and country level. A more resilient organization will undeniably enhance the wellbeing and prosperity of the community as well as sustaining normal business operations for the enterprise. 
This study has several limitations. The first limitation concerns the focus on the collective perception of employees based on the seven factors of commitment, communication, connection, coordination, community, consideration, and competency on organizational resilience and moving through the perception of ISA. This study was unable to incorporate the other dimension of perceived role clarity, the structure of working in the organization, and the response system developed to address organizational challenges both internally and externally. Therefore, future study should address these factors to gauge resilience. Additionally, future studies should measure the impact of ISA on a range of domains in light of organizational resilience across SMEs.

Secondly, this study was based on a cross-sectional design approach, and the capacity for resilience is incremental. Future research could adopt a longitudinal study design approach to explore organizational resilience as a developmental phenomenon based on the collective perception of the employees and also facilitated by the ISA utilized by the organization [3]. This study attempted to eliminate the single-source bias by informing the respondents about the anonymous nature of reporting of findings. Nevertheless, it is recommended that future studies should collect data from multiple sources in order to validate the findings. Notwithstanding, management of the organization should adopt a developmental perspective of the organization by examining the process of resilience by evaluating the collective stock of employees and enhancing the role of the ISA in the organization in order to achieve the goals and objectives of organizational resilience. Moreover, the issue of endogeneity must be addressed by applying the right strategy and approach in testing the issue in organizational resilience studies, including the treatment of endogeneity through introducing the control variable of the ex-post assessment with an input variable [47]. Future studies can run complementary methods to establish whether or not the issue of endogeniety is critical for the study results [45].

Author Contributions: S.R.V. prepared the research proposal, design the questionnaire, collected data and prepared the first draft. The remaining authors contributed developing the research model, literature review, sample selection, finalizing the questionnaire, sampling method, data analysis and finalizing the manuscript.

Funding: This research received no external funding.

Conflicts of Interest: The authors declare no conflict of interest.

\section{Appendix A}

Table A1. Survey Questionnaire.

\begin{tabular}{|c|c|}
\hline Code & Questions \\
\hline Commitment. Item-1 & I am focused and able to respond to the unexpected. \\
\hline Commitment. Item-2 & I am committed to take problem as my own and solve it. \\
\hline Commitment. Item-3 & I enjoy discussing my job and roles with other people to gain better ideas. \\
\hline Commitment. Item-4 & I have a strong sense of belonging among peers during adversity. \\
\hline Commitment. Item-5 & My organization culture motivates me to learn from success and failure for a more effective solution. \\
\hline Communication. Item-1 & I use effective messages that will raise awareness and build stability in handling crisis. \\
\hline Communication. Item-2 & $\begin{array}{l}\text { I am informed and updated so that I am able to disseminate more embedded knowledge to my } \\
\text { work groups. }\end{array}$ \\
\hline Communication. Item-3 & I follow the corporate culture and able to achieve amicable resolution during adversity. \\
\hline Communication. Item-4 & I am able to access critical information and communicate well to seek expert opinions. \\
\hline Communication. Item-5 & I am able to communicate openly across employees toward handling crisis and creating stability. \\
\hline Community. Item-1 & I work together to handle tasks efficiently and manage ambiguity. \\
\hline Community. Item-2 & I am committed to share more knowledge with staffs to solve problem. \\
\hline Community. Item-3 & I am able to get more support with relevant information from my workgroup for rebounding. \\
\hline Community. Item-4 & I am more enthusiastic about a problem until it is resolved. \\
\hline Community. Item-5 & We as a team feel responsible to address disruption that affects organizational effectiveness. \\
\hline Competency. Item-1 & I can stay focused and have a clear picture of what my roles would be under pressure. \\
\hline Competency. Item-2 & I am able to look at a situation from a different angle and able to solve problems. \\
\hline
\end{tabular}


Table A1. Cont.

\begin{tabular}{|c|c|}
\hline Code & Questions \\
\hline Competency. Item-3 & I resolve crisis competently at work. \\
\hline Competency. Item-4 & I re-evaluate my job and continuously improve the way I do my work. \\
\hline Competency. Item-5 & I take responsibility to fix problems competently. \\
\hline Connection. Item-1 & I have tight network connection view across employees and sector groups. \\
\hline Connection. Item-2 & $\begin{array}{l}\text { I am able to have close relationship with others in the industry to have better support and to take } \\
\text { appropriate action during adversity. }\end{array}$ \\
\hline Connection. Item-3 & I am able to work in a team to produce solutions that are more effective. \\
\hline Connection. Item-4 & I am able to share my strategies with peers and partners to avoid adversity. \\
\hline Connection. Item-5 & I am conscious about how crisis in our organization would affect other organizations. \\
\hline Coordination. Item-1 & $\begin{array}{l}\text { I often think about what could go wrong so that I can create ways to coordinate those } \\
\text { challenges efficiently. }\end{array}$ \\
\hline Coordination. Item-2 & I am able to analyze and negotiate with staffs to handle situations efficiently during crisis. \\
\hline Coordination. Item-3 & I coordinate my task effectively that enables to identify and analyze vulnerabilities. \\
\hline Coordination. Item-4 & I feel that I can handle many things at one time with simplified process. \\
\hline Consideration. Item-1 & In my organization, we set priorities for recovery from crisis. \\
\hline Consideration. Item-2 & $\begin{array}{l}\text { When I am struggling to accomplish something difficult, I focus on my progress instead of } \\
\text { feeling discouraged. }\end{array}$ \\
\hline Consideration. Item-3 & I am motivated to handle my job in an action-oriented approach in handling crisis. \\
\hline Consideration. Item-4 & I am able to make decisions on the basis that most of the up-to-date information will solve problem. \\
\hline Consideration. Item-5 & $\begin{array}{l}\text { I am able to respond to unexpected events and trigger for self-evaluation to improve the way I do } \\
\text { my work. }\end{array}$ \\
\hline I.S. Artifact. Item-1 & With the use of ISA, it is easy to find what I am looking for on my corporate site. \\
\hline I.S. Artifact. Item-2 & ISA could provide easy navigation of a task. \\
\hline I.S. Artifact. Item-3 & ISA offers a logical layout that is easy to follow. \\
\hline I.S. Artifact. Item-4 & With the use of ISA, function tasks would be integrated easily. \\
\hline I.S. Artifact. Item-5 & $\begin{array}{l}\text { ISA should reduce rigidity of response through less reliance on rigid use of existing procedures to } \\
\text { support organizational resilience. }\end{array}$ \\
\hline I.S. Artifact. Item-6 & With the use of ISA, I would be able to accomplish tasks more quickly. \\
\hline I.S. Artifact. Item-7 & With the use of ISA, I can easily complete my job. \\
\hline I.S. Artifact. Item-8 & ISA would improve my job performance. \\
\hline I.S. Artifact. Item-9 & With the use of ISA, my productivity in the job would increase. \\
\hline I.S. Artifact. Item-10 & ISA would be useful in my job. \\
\hline I.S. Artifact. Item-11 & My job would be effective with the use of ISA. \\
\hline I.S. Artifact. Item-12 & ISA should increase cognitive absorption and be able to block out most other distractions. \\
\hline I.S. Artifact. Item-13 & $\begin{array}{l}\text { ISA should increase cognitive absorption with features that enable focused immersion in whatever I } \\
\text { am doing. }\end{array}$ \\
\hline I.S. Artifact. Item-14 & ISA should increase cognitive absorption with features absorbed in what I am doing. \\
\hline I.S. Artifact. Item-15 & ISA should increase cognitive absorption with features that enable me to enjoy using the system. \\
\hline I.S. Artifact. Item-16 & ISA should reduce silo mentality that is detrimental to the resilience of employees and organizations. \\
\hline O. Resilience. Item-1 & My organization should respond in a resilient manner with the use of ISA. \\
\hline O. Resilience. Item-2 & My organization would be able to make positive adjustment under challenging conditions. \\
\hline O. Resilience. Item-3 & My organization should bounce back fast and achieve resilience. \\
\hline O. Resilience. Item-4 & My organization would adapt positively in a situation of significant adversity. \\
\hline O. Resilience. Item-5 & Using ISA, my organization is capable of decreasing vulnerability effectively. \\
\hline O. Resilience. Item-6 & Using ISA, my organization develops alternatives in order to benefit from negative circumstances. \\
\hline
\end{tabular}

\section{References}

1. Mallak, L.A.; Yildiz, M. The Development of a Workplace Resilience. J. Prev. Assess. Rehabil. 2016, 54, 241-253.

2. Annarelli, A.; Nonino, F. Strategic and operational management of organisational resilience: Current state of research and future directions. Omega 2016, 62, 1-18. [CrossRef] 
3. Linnenluecke, M.K. Resilience in Business and Management Research: A Review of Influential Publications and a Research Agenda. Int. J. Manag. Rev. 2017, 19, 4-30. [CrossRef]

4. Burnard, K.; Bhamra, R. Organisational resilience: Development of a conceptual framework for organisational responses. Int. J. Prod. Res. 2011, 49, 5581-5599. [CrossRef]

5. Burns, A.J.; Posey, C.; Roberts, T.L.; Benjamin, L.P. Examining the relationship of organisational insiders' psychological capital with information security threat and coping appraisals. Comput. Hum. Behav. 2017, 68, 190-209. [CrossRef]

6. Buckle, P. Assessing social resilience. In Disaster Resilience: An Integrated Approach; Paton, D., Johnston, D., Eds.; Charles C. Thomas: Springfield, IL, USA, 2006.

7. Horne, J.F.; Orr, J.E. Assessing behaviours that create resilient organisations. Employ. Relat. Today 1988, 24, 29-39.

8. Lee, A.V.; Vargo, J.; Seville, E. Developing a tool to measure and compare organisations' resilience. Nat. Hazards Rev. 2013, 14, 29-41. [CrossRef]

9. Connor, K.M.; Davidson, J.R. Development of a new resilience scale: The Connor-Davidson resilience scale (CR-RISC). Depress. Anxiety 2003, 18, 76-82. [CrossRef]

10. Mafabi, S.; Munene, J.C.; Ahiauzu, A. Creative climate and organisational resilience: The mediating role of innovation. Int. J. Organ. Anal. 2015, 23, 564-587. [CrossRef]

11. Manyena, S.B. The conpcet of resiliecne revisted. Disasters 2006, 30, 434-450. [CrossRef]

12. McManus, S.; Seville, E.; Vargo, J.; Brunsdon, D. A facilitated process for improving organisational resilience. Nat. Hazards Rev. 2008, 9, 81-90. [CrossRef]

13. Bunderson, J.S.; Lofstrom, S.M.; Van De Ven, A.H. Conceptualizing and measuring professional and administrative models of organizing. Organ. Res. Methods 2000, 3, 366-391. [CrossRef]

14. Luthans, F. The need for and meaning of positive organisational behaviour. J. Organ. Behav. 2002, 23, 695-706. [CrossRef]

15. Youssef, C.M.; Luthans, F. Positive Organisational Behavior in the Workplace: The Impact of Hope, Optimism, and Resilience. J. Manag. 2007, 33, 774-800.

16. Mallak, L.A. Measuring resilience in health care provider organisations. Health Manpow. Manag. 1998, 24, 148-152. [CrossRef]

17. Smith, D. Beyond contingency planning: Towards a model of crisis management. Ind. Crisis Q. 1990, 4, 263-275. [CrossRef]

18. Hollnagel, E. How Resilient Is Your Organisation? An Introduction to the Resilience Analysis Grid (RAG). 2010. Available online: https://hal-mines-paristech.archives-ouvertes.fr/hal-00613986/document (accessed on 6 June 2019).

19. Powley, E.H. Reclaiming resilience and safety: Resilience activation in the critical period of crisis. Hum. Relat. 2009, 62, 1289-1326. [CrossRef]

20. Gilly, J.P.; Kechidi, M.; Talbot, D. Resilience of organisations and territories: The role of pivot firms. Eur. Manag. J. 2014, 32, 596-602. [CrossRef]

21. Ruiz-Martin, C.; López-Paredes, A.; Wainer, G. What we know and do not know about organisational resilience. Int. J. Prod. Manag. Eng. 2018, 6, 11-28. [CrossRef]

22. Somers, S. Measuring resilience potential: An adaptive strategy for organisational crisis planning. J. Contingencies Crisis Manag. 2009, 17, 12-23. [CrossRef]

23. Wing, S.C.; Wai, O.H. Determinants of the critical success factor of disaster recovery planning for information systems. Inf. Manag. Comput. Secur. 2009, 17, 248-275.

24. Sheffi, Y. Building a Resilient Organisation. 2006. Available online: http://web.mit.edu/sheffi/www/ selectedMedia/wpd_building_a_resilient_organization_rev20061226.pdf (accessed on 6 June 2019).

25. Erol, O.; Sauser, B.J.; Mansouri, M. A framework for investigation into extended enterprise resilience. Enterp. Inf. Syst. 2010, 4, 111-136. [CrossRef]

26. Gunasekaran, A.; Rai, B.K.; Griffin, M. Resilience and competitiveness of small and medium-size enterprises: An empirical research. Int. J. Prod. Res. 2011, 49, 5489-5509. [CrossRef]

27. Fischbacher-Smith, D.; Fischbacher-Smith, M. The changing nature of risk and risk management: The challenge of borders, uncertainty and resilience. Risk Manag. 2009, 11, 1-12. [CrossRef]

28. Lengnick-Hall, C.A.; Beck, T.E.; Lengnick-Hall, M.L. Developing a capacity for organisational resilience through strategic human resource management. Hum. Resour. Manag. Rev. 2011, 21, 243-255. [CrossRef] 
29. Saade, R.; Bahli, B. The impact of cognitive absorption on perceived usefulness and perceived ease of use in on-line learning: An extension of the technology acceptance model. Inf. Manag. 2005, 42, 317-327. [CrossRef]

30. Zellars, K.L.; Justice, L.; Beck, T.E. Resilience: New paths for building and sustaining individual and organisational capacity. In Research in Occupational Stress and Well Being; Perrewé, P., Halbesleben, J., Rosen, C., Eds.; Emerald Publishing Limited: Bingley, UK, 2011; Volume 9, pp. 1-37.

31. Liisa, V.A.; Romme, G.L. Building resilience capabilities at “Big Brown Box, Inc.". Strategy Leadersh. 2012, 40, 43-45.

32. Wicker, P.; Filo, K.; Cuskelly, G. Organisational resilience of community sport clubs impacted by natural disasters. J. Sport Manag. 2013, 27, 510-525. [CrossRef]

33. Whitman, Z.R.; Kachali, H.; Roger, D.; Vargo, J.; Seville, E. Short-form version of the Benchmark Resilience Tool (BRT-53). Meas. Bus. Excell. 2013, 17, 3-14. [CrossRef]

34. Riolli, L.; Savicki, V. Information system organisational resilience. Omega 2003, 31, 227-233. [CrossRef]

35. Venkatesh, V. Determinants of perceived ease of use: Integrating control, intrinsic motivation, and emotion into the technology acceptance model. Inf. Syst. Res. 2000, 11, 342-365. [CrossRef]

36. Allen, N.J.; Meyer, J.P. The measurement and antecedents of affective, continuance and normative commitment to the organisation. J. Occup. Organ. Psychol. 1990, 63, 1-18. [CrossRef]

37. Al-Natour, S.; Benbasat, I. The adoption and use of IT artefacts: A new interaction-centric model for the study of user-artefact relationship. J. Assoc. Inf. Syst. 2009, 10, 661-685.

38. Chin, W.W. How to write up and report PLS analyses. In Handbook of Partial Least Squares; Vinzi, V.E., Chin, W.W., Henseler, J., Wang, H., Eds.; Springer: Berlin, Germany, 2010.

39. Podsakoff, P.M.; MacKenzie, S.B.; Lee, J.-Y.; Podsakoff, N.P. Common method biases in behavioural research: A critical review of the literature and recommended remedies. J. Appl. Psychol. 2003, 88, 879-903. [CrossRef] [PubMed]

40. Harman, H.H. Modern Factor Analysis, 3rd ed.; The University of Chicago Press: Chicago, IL, USA, 1976.

41. Peng, D.X.; Lai, F. Using partial least squares in operations management research: A practical guideline and summary of past research. J. Oper. Manag. 2012, 30, 467-480. [CrossRef]

42. Cain, M.K.; Zhang, Z.; Yuan, K.-H. Univariate and multivariate skewness and kurtosis for measuring nonnormality: Prevalence, influence and estimation. Behav. Res. Methods 2017, 49, 1716-1735. [CrossRef]

43. Hair, J.F.; Ringle, C.M.; Sarstedt, M. Editorial-partial least squares structural equation modeling: Rigorous applications, better results and higher acceptance. Long Range Plan. 2014, 46, 1-12. [CrossRef]

44. Cohen, J. Statistical Power Analysis for the Behavioral Sciences, 2nd ed.; Lawrence Earlbaum Associates: Hillsdale, NJ, USA, 1988.

45. Sarstedt, M.; Ringle, C.M.; Cheah, J.H.; Ting, H.; Moisescu, O.I.; Radomir, L. Structural model robustness check in PLS-SEM. Tour. Econ. 2019, 1-24. [CrossRef]

46. Park, S.; Gupta, S. Handling Endogenous Regressors by Joint Estimation Using Copulas. Mark. Sci. 2012, 31 , 567-586. [CrossRef]

47. Hult, G.T.M.; Hair, J.F.; Proksch, D.; Sarstedt, M.; Pinkwart, A.; Ringle, C.M. Addressing endogeneity in international marketing applications of partial least squares structural equation modeling. J. Int. Mark. 2018, 26, 1-21. [CrossRef]

(C) 2019 by the authors. Licensee MDPI, Basel, Switzerland. This article is an open access article distributed under the terms and conditions of the Creative Commons Attribution (CC BY) license (http://creativecommons.org/licenses/by/4.0/). 